\title{
Hydrophobic Hydration of Fluoroalkyl (C-F) is Distinctly Different from that of Its Hydrogenated Counterpart (C-H) as Observed by Raman Difference with Simultaneous Curve Fitting Analysis
}

Subhadip Roy ${ }^{\dagger}$, Biswajit Biswas ${ }^{\dagger+}$, Nishith Ghosh ${ }^{\ddagger}$, P. C. Singh ${ }^{++*}$, Jahur A. Mondal $^{+*}$

${ }^{+}$Radiation \& Photochemistry Division, Industrial Hygiene \& Safety Section Bhabha Atomic Research Centre, Homi Bhabha National Institute, Trombay, Mumbai 400085, India

${ }^{+}$School of Chemical Sciences, Indian Association for the Cultivation of Science, Kolkata 700032, India

*mondal@barc.gov.in; sppcs@iacs.res.in

\section{Supporting Information.}

Raman difference with simultaneous curve fitting (RD-SCF) Analysis. In RD-SCF analysis, the experimental Raman spectrum of diluted alcohol solution is modelled as a linear combination of two spectral components: (1) spectrum of the bulk water, which is actually the major component and (2) the spectrum of the hydration shell water along with the vibrational modes of alcohol (e.g. $\mathrm{CH}$ and alcoholic-OH stretches). Because of low concentration of alcohol, the component pertaining to the hydration shell water (of the alcohol) is the minor component. The purpose of the "differential" and "curve fitting" analyses (RD-SCF) is to extract the minor component (i.e. hydration water spectrum along with the intramolecular modes of the solute called as solute correlated solvent spectrum; SC-spectrum) which is buried in an undesired major component (bulk water spectrum). In this context, Raman multivariate curve resolution (Raman-MCR) analysis is a powerful technique, which is pioneered by BenAmotz and coworkers. ${ }^{1-4}$ to extract the minimum area SC-spectrum of hydrophobes and ions in water (hydration shell spectrum). In fact, we also have used Raman-MCR to extract SCspectra for ions, hydrophobes and metabolites that revealed vibrational perturbation of water in the hydration shell of the solutes. ${ }^{5-8}$ The strength of Raman-MCR lies with the fact that it does not need any assumed fitting function while extracting the minimum area SC-spectrum. Minimum area SC-spectrum means, the bulk-water-like spectral response has been removed from the solute associated spectrum to the maximum possible extent until there appears a point of zero signal within the region of vibrational resonance of the $\mathrm{OH}$ stretch $(\sim 3000-3700$ $\mathrm{cm}^{-1}$ ). As a result, in the case of two component system, the MCR-extracted hydration shell spectrum is essentially the minimum area difference spectrum, which can also be obtained by 
simple subtraction of the bulk water spectrum from the solution spectrum. The minimum area SC-spectrum (or minimum area difference spectrum) certainly reveals the spectral features of hydration water; however, it may not always correspond to the true band shape of the spectrum of the hydration shell water. Moreover, the quantitative information, for example, the number of water molecules affected by a particular solute molecule, cannot be directly obtained from the minimum area SC-spectrum, as the absolute intensity information is not retained in the SCspectrum. To overcome these limitations, we applied a different analytical approach, which is similar to the double difference IR (DDIR) spectroscopy developed by Lindgren and coworkers, ${ }^{9}$ which they applied in aqueous electrolyte solutions to extract cation- and anionaffected water by further fitting analysis of the DDIR-spectrum. In RD-SCF, we model the Raman spectrum of dilute alcohol solution $\left(S(\omega)_{\text {exp }}\right)$ by simultaneous curve fitting with two inputs: (1) multicomponent Gaussian bands and (2) the experimentally obtained spectrum of the bulk water $\left(R(\omega)_{\text {exp }}\right.$; as a reference for the solute-unaffected water) as shown in the following equation 1 .

$$
S(\omega)_{f i t}=\sum_{n=1}^{4}\left(a_{n} \exp \left(-(\omega-\omega n)^{2} / 2 \Gamma_{n}^{2}\right)+f R(\omega)_{e x p}+a_{0}\right.
$$

Where, $f$ is the fraction of the bulk water spectrum $\left(R(\omega)_{\text {exp }}\right)$ contained in the sample spectrum $\left(S(\omega)_{\text {exp }}\right) ; a_{n}, \omega n, \Gamma n$ are the amplitude, centre frequency and fwhm of the nth component band of the multi-component fitted curve, $a_{0}$ is a constant, which takes care of the constant background difference (if any) between the spectra of the sample and reference. To have a physically meaningful band-shapes of the components, all the coefficients were restricted to be nonnegative during the optimization of the fitted spectrum using iterative non-linear least square method, such that the $\chi_{\text {square }}$ value, defined by equation (2), is minimized.

$$
\chi_{\text {square }}=\sum_{i}\left(\frac{S(\omega i)_{\exp }-S(\omega i)_{f i t}}{\sigma\left(\omega_{i}\right)}\right)^{2}
$$

Where, $S(\omega i)_{\text {exp }}$ is the measured Raman intensity at a given wavenumber, $\omega i$ and $S(\omega i)_{f i t}$ is the fitted intensity at that wavenumber; and $\sigma\left(\omega_{i}\right)$ is the standard deviation for $S\left(\omega_{i}\right)_{f i t}$. Thus, in the RD-SCF analysis, the value of the coefficient $f$, which governs the amount of bulk water needs to be removed to recover the SC-spectrum $\left(S_{H S}\right)$ is optimized by simultaneous fitting of the solution spectrum with Gaussian bands and the reference spectrum (bulk water spectrum). 
The spectrum of the hydration shell water $\left(S(\omega)_{H S}\right)$, which represents the true "band shape" and "intensity" of the solute-associated solvent can be expressed as,

$S(\omega)_{H S}=S(\omega)_{\exp }-f R(\omega)_{\exp }-a_{0}$

Like any other fitting analysis, the success of RD-SCF also lies with proper choice of number components and an appropriate initial estimate of their coefficients. In this context, it is important to note that the number of Gaussian components introduced in the fitting analysis and the initial guess of their corresponding coefficients are not chosen arbitrarily for RD-SCF. Rather, they are guided by the band shape of the Raman spectrum the bulk water (reference) and that of the sample (in case, the sample also contains vibrational bands in the spectral region of interest). Since, the hydration shell water is basically "perturbed bulk water" (solute-affected solvent), the spectrum of the hydration water is expected to be a kind of perturbed spectrum of the bulk water. The same is true for the solute (solvent-affected solute spectrum). On the basis of this assumption, the coefficients of the fitted curve of the neat solvent (water) and solute (alcohol) spectra with least number of Gaussian bands (for the water $\mathrm{OH}$ stretch band, it is four ${ }^{10}$ ) provides a good initial estimation of coefficients that are fed into the analysis as initial guess. The SC-spectrum extracted by this method is highly reproducible. Quite interestingly, for the alcohol-water system studied here, the band shape of the peak normalized ( $\mathrm{CH}$ stretch) RD-SCF-extracted spectrum is quite similar to the Raman-MCR spectrum, though there is a little difference in intensity in the $\mathrm{OH}$ stretch region (Figure S1). Thus, for two component system, such as the alcohol-water mixture, where the spectrum of one of the pure component (bulk water) is known, RD-SCF provides not only the more realistic spectrum of the hydration shell water, but also the number of water molecules affected by a solute (intensity information). Using RD-SCF, we demonstrate that fluorination of an alkyl group (molecular hydrophobe) leads to remarkable change in the hydrophobic hydration.

\section{Comparison of the hydration shell spectra extracted by RD-SCF and Raman-MCR}

analyses. Ethanol as well as isopropanol correlated water spectra extracted by RD-SCF (solid curves) and Raman-MCR (dashed curves) analysis are shown in Figure S1a and S1b. The actual intensity of RD-SCF extracted spectrum is higher than that of the Raman-MCR. To compare the band shape in the $\mathrm{OH}$ stretch region, we presented the spectra by intensity normalization at the $\mathrm{CH}$ stretch maximum (Figure S1). For the alcohol-water system, the band shape in the $\mathrm{OH}$ stretch region is quite similar for both the analytical methods, though the zero point (zero signal 
at an wavenumber within the $\mathrm{OH}$ stretch region; dashed curve in inset Fig S1) at around 3630 $\mathrm{cm}^{-1}$ has not been observed for the Rd-SCF spectrum (solid curve in inset).
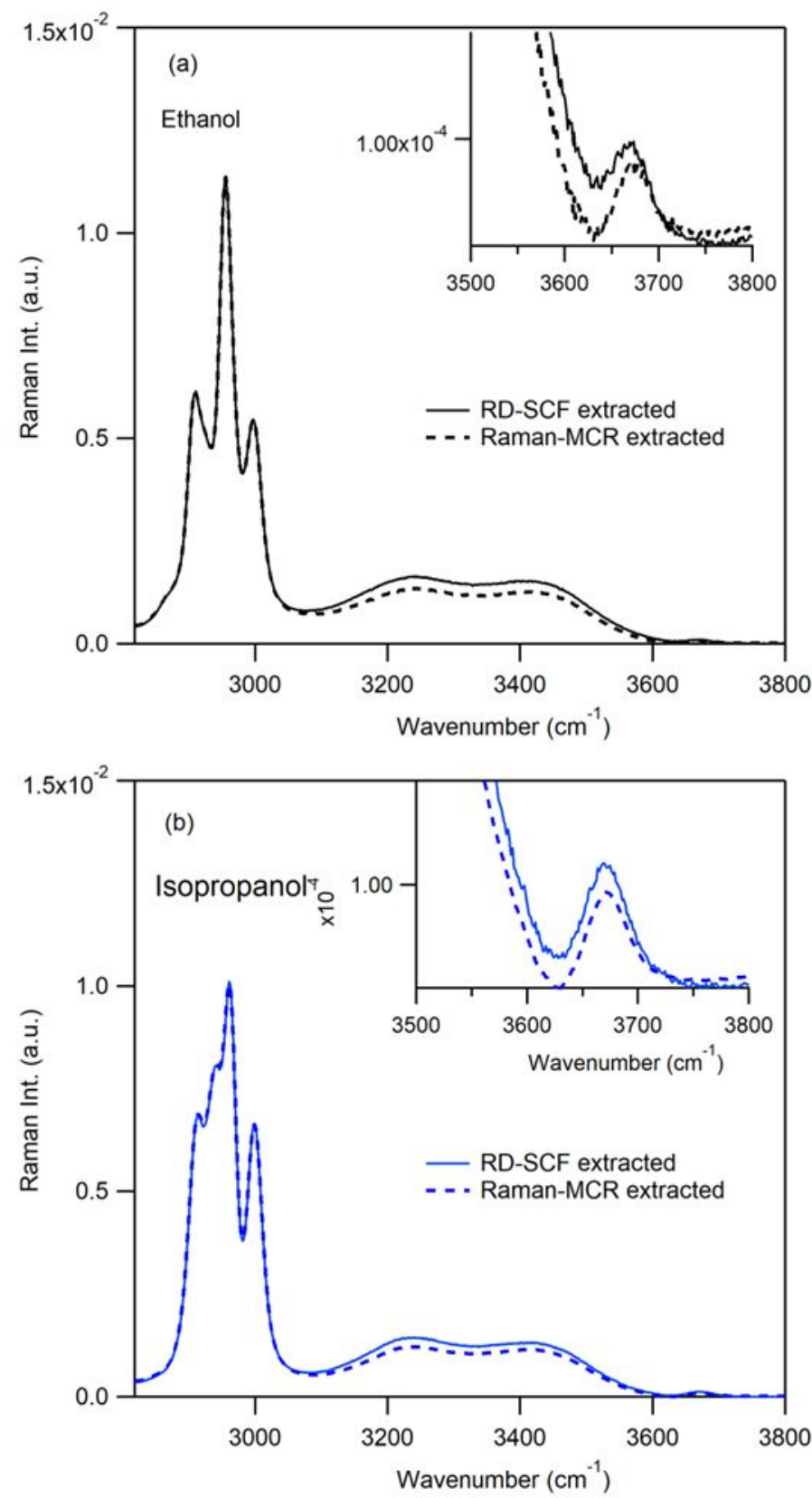

Figure S1. RD-SCF (solid curves) and Raman-MCR (dashed curves) extracted spectra of (a) ethanol and (b) isopropanol in the $\mathrm{CH}$ and $\mathrm{OH}$ stretch region $\left(2815-3800 \mathrm{~cm}^{-1}\right)$

Polarised and depolarised spectra of water in the hydration shell of ET and TFE. The polarized (i.e. parallel; $I_{\| I}$ ) and depolarized (i.e. perpendicular; $I_{\perp}$ ) Raman spectra of hydration shell water extracted from the corresponding Raman spectra of 0.5M ET and TFE solutions (Figure S2). The depolarization ratio, $\rho=\mathrm{I}_{\perp} / \mathrm{I}_{\| l}$, for the hydration water of ET and TFE and that 
of the bulk water are shown in Figure S2(c). The low value of the depolarization ratio indicates the polarized (symmetric) nature of $\mathrm{OH}$ stretch vibration of bulk water. In the hydration shell of ET as well, the $\mathrm{OH}$ stretch of water is largely polarized. However, in the case of TFE, the value of the depolarization ratio is quite high around $3500 \mathrm{~cm}-1$, indicating the depolarized nature i.e. asymmetrical H-bonding of water in the hydration shell of the latter. The variation of $\rho$ around 3600 is noisy (grey shaded region), because of very low intensity of either the polarized and/or the depolarized spectra in that region. Nevertheless, the $\rho$ values around 3670 $\mathrm{cm}^{-1}$ is quite reliable, and it is close to the central value of the depolarization ratio, which indicates that the dangling $\mathrm{OH}$ is largely decoupled (vibrationally) at the surface of the molecular hydrophobes. In other words, the dangling $\mathrm{OH}$ exists as an independent $\mathrm{OH}$ oscillator at the surface of both, alkyl and fluoroalkyl groups.
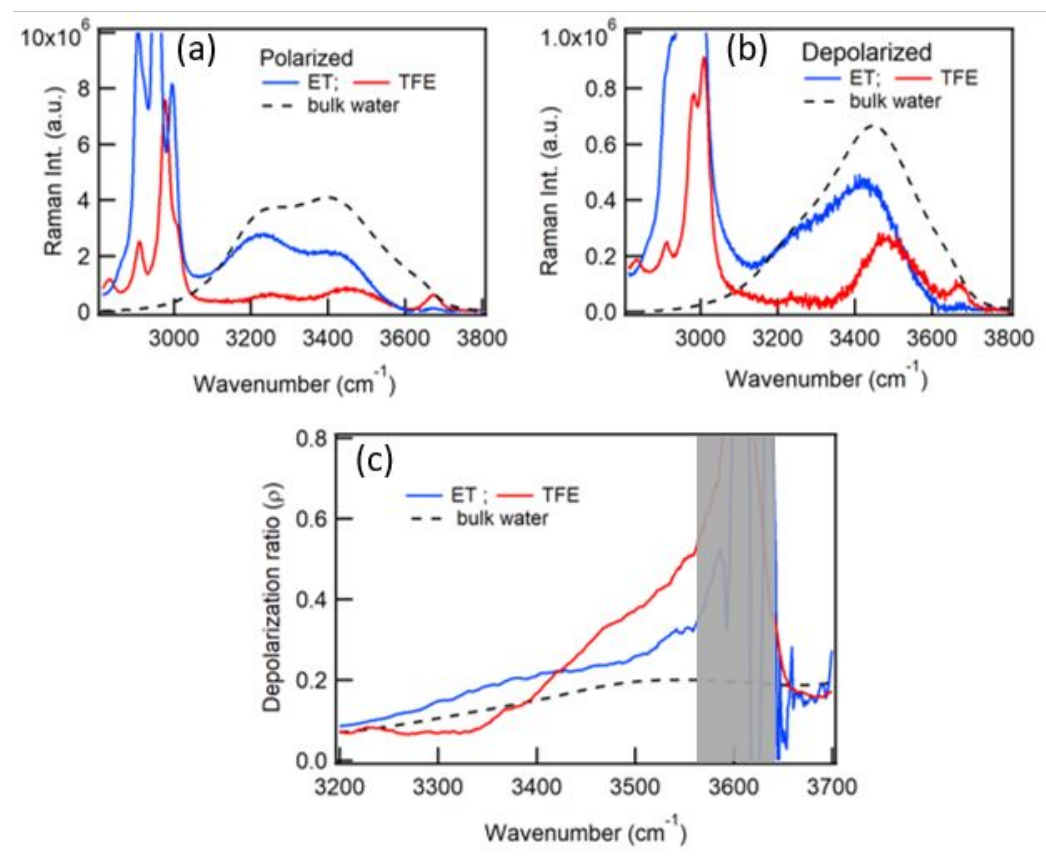

Figure S2. (a) Polarized (parallel) and (b) depolarized (perpendicular) Raman spectra of water in the hydration shell of ET (blue curve) and TFE (red curve). The depolarization ratio ( $\rho$ ) of the respective hydration shell are shown in panel (c). The corresponding spectrum of the bulk water (dashed curve) is shown for reference in each panel. 
Table S1. Three component Gaussian fitting parameters of alcohol associated water in the $\mathrm{OH}$ stretch region.

\begin{tabular}{|c|c|c|c|c|c|c|c|}
\hline $\begin{array}{c}\text { OH stretch } \\
\text { Region }\end{array}$ & Coefficients & ET & IP & MFE & DFE & TFE & HFIP \\
\hline \multirow{2}{*}{$\begin{array}{c}\text { peak } 1 \\
\left(\sim 3250 \mathrm{~cm}^{-1}\right)\end{array}$} & amplitude $\mathrm{x} 10^{-5}$ & 1.37 & 1.61 & 0.082 & 0.057 & 0.11 & 0.098 \\
\cline { 2 - 8 } & peak centre $\left(\mathrm{cm}^{-1}\right)$ & 3236 & 3236 & 3246 & 3272 & 3270 & 3271 \\
\cline { 2 - 8 } & fwhm $\left(\mathrm{cm}^{-1}\right)$ & 153 & 133 & 77 & 43 & 57 & 41 \\
\hline \multirow{2}{*}{$\begin{array}{c}\text { peak } 2 \\
\left(\sim 3450 \mathrm{~cm}^{-1}\right)\end{array}$} & amplitude $\times 10^{-5}$ & 1.04 & 1.32 & 0.33 & 0.46 & 0.43 & 0.56 \\
\cline { 2 - 8 } & peak centre $\left(\mathrm{cm}^{-1}\right)$ & 3442 & 3436 & 3461 & 3471 & 3470 & 3488 \\
\cline { 2 - 8 } & fwhm $\left(\mathrm{cm}^{-1}\right)$ & 102 & 102 & 85 & 112 & 100 & 134 \\
\hline \multirow{2}{*}{\begin{tabular}{c}
$\left(\sim 3670 \mathrm{~cm}^{-1}\right)$ \\
\cline { 2 - 8 }
\end{tabular}} & amplitude $\mathrm{x} 10^{-5}$ & 0.067 & 0.13 & 0.25 & 0.38 & 0.33 & 0.52 \\
\cline { 2 - 8 } & peak centre $\left(\mathrm{cm}^{-1}\right)$ & 3670 & 3673 & 3630 & 3660 & 3673 & 3675 \\
\hline
\end{tabular}

\section{References}

1. Fega, K. R.; Wilcox, D. S.; Ben-Amotz, D., Application of Raman Multivariate Curve Resolution to Solvation-Shell Spectroscopy. Appl. Spectrosc. 2012, 66, 282-288.

2. Wu, X.; Lu, W.; Streacker, L. M.; Ashbaugh, H. S.; Ben-Amotz, D., Methane Hydration-Shell Structure and Fragility. Angew. Chem. Int. Ed. 2018, 57, 15133-15137.

3. Davis, J. G.; Rankin, B. M.; Gierszal, K. P.; Ben-Amotz, D., On the Cooperative Formation of Non-Hydrogen-Bonded Water at Molecular Hydrophobic Interfaces. Nature Chem. 2013, 5, 796-802.

4. Davis, J. G.; Zukowski, S. R.; Rankin, B. M.; Ben-Amotz, D., Influence of a Neighboring Charged Group on Hydrophobic Hydration Shell Structure. J. Phys. Chem. B 2015, 119, 9417-9422.

5. Ahmed, M.; Namboodiri, V.; Mathi, P.; Singh, A. K.; Mondal, J. A., How Osmolyte and Denaturant Affect Water at the Air-Water Interface and in Bulk: A Heterodyne-Detected Vibrational Sum Frequency Generation (Hd-Vsfg) and Hydration Shell Spectroscopic Study. J. Phys. Chem. C 2016, 120, 10252-10260.

6. Ahmed, M.; Namboodiri, V.; Singh, A. K.; Mondal, J. A., On the Intermolecular Vibrational Coupling, Hydrogen Bonding, and Librational Freedom of Water in the Hydration Shell of Mono- and Bivalent Anions. J. Chem. Phys. 2014, 141, 164708.

7. Ahmed, M.; Namboodiri, V.; Singh, A. K.; Mondal, J. A.; Sarkar, S. K., How lons Affect the Structure of Water: A Combined Raman Spectroscopy and Multivariate Curve Resolution Study. J. Phys. Chem. B 2013, 117, 16479-16485.

8. Ahmed, M.; Singh, A. K.; Mondal, J. A., Hydrogen-Bonding and Vibrational Coupling of Water in a Hydrophobic Hydration Shell as Observed by Raman-Mcr and Isotopic Dilution Spectroscopy. Phys. Chem. Chem. Phys. 2016, 18, 2767-2775.

9. Kristiansson, O.; Eriksson, A.; Lindgren, J., Hydration of lons in Aqueous Solution Studied by Ir Spectroscopy. J. Mol. Struct. 1984, 114, 455-458.

10. Carey, D. M.; Korenowski, G. M., Measurement of the Raman Spectrum of Liquid Water. J. Chem. Phys. 1998, 108, 2669-2675. 